\title{
EFFICACITE DES TRAITEMENTS PHYTOSANITAIRES CONTRE LES RAVAGEURS ET MALADIES DE QUATRE VARIETES DE TOMATE AU CENTRE DE LA CÔTE D'IVOIRE
}

\author{
K. ADJE, A. H. DJIDJI, L. FONDIO, J. C. N'ZI et C. KOUAME \\ Centre National de Recherche Agronomique (CNRA) Programmes Cultures Maraîchères et Protéagineuses. \\ 01 BP 1740 Abidjan 01
}

\begin{abstract}
RESUME
L'évolution des populations d'insectes ravageurs et des maladies, ainsi que leur incidence sur la récolte et l'efficacité des traitements chimiques ont été étudiées à la Station Expérimentale du Foro-Foro de l'Institut des Savanes (ex-IDESSA). Deux fongicides Manate $80 \%$ et Peltar Flo et deux insecticides Décis 12 EC (deltaméthrine) et Systoate (dimethoate) ont été expérimentés en 9 combinaisons de traitements sur quatre variétés de tomate (Rossol, Petomech, Avinash-2 et IDSA07-390). Les observations et mesures ont porté principalement sur le niveau des populations et l'incidence des mouches blanches, des chenilles, des maladies fongiques et virales et sur le rendement de quatre variétés de tomate. L'étude a montré que la deltaméthrine et le diméthoate sont efficaces contre les mouches blanches et les chenilles. Quant aux chenilles, la noctuelle (Spodoptera sp.) s'avère bien plus importante aussi bien par le nombre de chenilles que par les dégâts causés sur les fruits. Les maladies mycologiques observées en végétation ont révélé, principalement, les pourritures du collet, les dégâts d'alternariose et de stemphyliose. Le manèbe semble bien contrôler ces maladies. Les pertes de récoltes dues aux insectes et champignons ont été évaluées à $23,7 \%$ et $32,5 \%$. Le traitement des parcelles à la deltaméthrine et au manèbe a induit des gains nets de production entre 160 et $180 \%$.
\end{abstract}

Mots clés : Fongicides, insecticides, ravageurs, maladies, tomate, Solanum lycopersicum L.

\begin{abstract}
INSECTS PESTS AND DISEASES OF FOUR TOMATOES VARIETIES IN CENTRAL CÔTE D'IVOIRE AND EFFECT OF SOME CULTURAL PRACTISES
\end{abstract}

Population evolution of devastating insects and diseases as well as their incidence on harvest and the efficacity of chemical treatments were tried on tomato (Solanum lycopersicum L.) at IDESSA Research Station of Foro-Foro. The efficacities of two fungicides Manate $80 \%$ and Peltar Flo and two insecticides Decis 12 EC (deltamethrine) and Systoate (dimethoate) on tomato pests were studied in a combinations of a treatments, using 4 tomatoes varieties (Rossol, Petomech, Avinash-2 and IDSA 97-370). Data were collected on insect pest population dynamic, fungal and viral diseases incidence and yield of the 4 tomato varieties. The study showed that both tested insecticides deltamethrine and dimethoate effectively controled the population of whiteflies and caterpillars. Caterpillars number of nocturnal (Spodoptera sp.) were more important than their damage on tomato fruit. Fungal diseases, observed in field, showed, mainly, snare rot, damage of alternariose and stemphyliose. The Manate $80 \%$ seems to control well these diseases. Yield loss caused by insect pests and fungal diseases were evaluated to $23.7 \%$ and $32.5 \%$, respectively. Combined treatment of deltamethrine and manebe increased the net gain of production yield of tomato by 160 to $180 \%$.

Keys words: Fungicide, insecticide, insect pests, Diseases, tomato, Solanum lycopersicum L. 


\section{INTRODUCTION}

La tomate (Solanum lycopersicum L.) est l'un des légumes les plus cultivés dans le monde. Originaire d'Amérique du Sud, l'extension de la culture de la tomate dans tous les continents, sous plusieurs latitudes, a entraîné une multiplication des problèmes phytosanitaires. Ainsi, plus de 200 maladies ont-elles été décrites sur la tomate (Philouze et Laterrot, 1992). En Côte d'Ivoire, comme dans la plupart des pays tropicaux, la culture de la tomate est soumise à plusieurs contraintes biotiques et abiotiques. Les fortes températures et l'hygrométrie de ces régions a entraîné une prolifération des maladies, causant une baisse significative de la production et une détérioration de la qualité du fruit (Anonyme, 1977 ; Conover et Gerhald, 1981 ; Nechadi et al., 2002 ; Open et al., 1989 et Pohronezny et Valin, 1983).

L'amélioration de la productivité de la tomate reste cependant un impératif de développement en Côte d'Ivoire. La demande en tomate, sans cesse croissante aussi bien pour la consommation en frais que pour la transformation industrielle est supérieure à l'offre. La culture de variétés adaptées et productives, ainsi que la maîtrise des facteurs de production, dont le contrôle judicieux des maladies et des insectes ravageurs, s'avèrent indispensables. Cette étude vise d'une part, à déterminer les périodes d'infestation et de pullulation intenses des insectes ravageurs et des maladies de la tomate au centre de la Côte d'Ivoire et, d'autre part, à évaluer l'efficacité de quelques produits phytosanitaires commer-cialisés localement.

\section{MATERIEL ET METHODES}

Cette étude a été conduite à la station expérimentale de Foro-Foro de l'ex-Institut des Savanes de Bouaké Côte d'Ivoire $\left(7^{\circ} 46 \mathrm{~N}, 5^{\circ} 06\right.$ W). Quatre variétés de tomate (Solanum lycopersicum L.) ont été évaluées au cours de cet essai ; il s'agit de : Rossol, petomech, Avinash-2, IDSA 97-370 qui ont été semées le
2 octobre 1995. Deux insecticides, Decis 12 EC ( $12 \mathrm{~g}$ de deltaméthrine / litre) et Systoate $40 \mathrm{EC}$ ( $400 \mathrm{~g}$ de diméthoate / litre) et deux fongicides, Manate $80 \%$ ( $800 \mathrm{~g}$ de manèbe / litre) et Peltar (150 g de métylthiophanate / litre) et un témoin non traité, ont été expérimentés dans une combinaison de 9 traitements (Tableau 1). L'essai a été disposé selon un split-plot avec un arrangement aléatoire des traitements. La variété a constitué le facteur principal et le traitement phytosanitaire, le facteur secondaire. Trois répétitions ont été effectuées.

Les plants ont été conduits en pépinière avant d'être transplantées au champ 21 jours plus tard. Le repiquage a été réalisé en doubles lignes espacées de $0,50 \mathrm{~m}$ sur des billons. La distance entre les poquets a été de $0,40 \mathrm{~m}$ avec des parcelles élémentaires de $8 \mathrm{~m}^{2}$. Un épandage d'engrais de fond (NPK 10-18-18) à la dose de $300 \mathrm{~kg} / \mathrm{ha}$ a été fait avant repiquage. Deux apports d'urée $(100 \mathrm{~kg} / \mathrm{ha})$ et de sulfate de potasse $(200 \mathrm{~kg} / \mathrm{ha})$ ont été effectués au $15^{\mathrm{e}}$ jour après repiquage. Les traitements phytosanitaires ont été réalisés 6 fois au total, à 15 jours d'intervalle après repiquage, à l'aide d'un pulvérisateur manuel. Le diméthoate étant un produit systémique, son application a été arrêtée à partir de la floraison et remplacée par la deltaméthrine.

Les mesures expérimentales ont porté sur le comptage des insectes (mouche blanche et chenilles), la sévérité des maladies et dégâts de ravageurs, et les rendements. La sévérité des symptômes a été notée sur une échelle de 1 à $5(1=$ faible, $3=$ moyen, $5=$ très sévère $)$. Pour le comptage des insectes, 5 plants ont été choisis au hasard dans chaque parcelle. Les insectes ont été collectés une fois par semaine. A chaque récolte, aussi bien les fruits avariés et les fruits commercialisables ont été comptés et pesés. Les rendements et les pourcentages d'avarie ont été calculés. Les données ont été analysées suivant le modèle linéaire du logiciel SAS (SAS Institute, 1986). Les moyennes ont été séparées selon le test de Duncan au seuil de $5 \%$. 
Tableau 1 : Combinaison de fongicide et d'insecticide testés.

Experimental treatment used in study.

\begin{tabular}{llll}
\hline $\mathrm{N}^{\circ}$ & Traitements & Produits commerciaux & Matières actives \\
\hline 1 & I0F0 & Témoin non traité & --- \\
\hline 2 & I1F0 & Decis 12 EC & deltaméthrine \\
\hline 3 & I2F0 & Systoate $400 \mathrm{EC}$ & diméthoate \\
\hline 4 & I0F1 & Manate 80 & manèbe \\
\hline 5 & I1F1 & Decis $12 \mathrm{EC}+$ Manate 80 & deltaméthrine + manèbe \\
\hline 6 & I2F1 & Systoate 400 EC + Manate 80 & diméthoate + manèbe \\
\hline 7 & I0F2 & Peltar & methylthiophanate \\
\hline 8 & I1F2 & Decis 12 EC + Peltar & deltaméthrine + methylthiophanate \\
\hline 9 & I2F2 & Systoate 400 EC + Peltar & diméthoate + methylthiophanate
\end{tabular}

$\mathrm{I}=$ Insecticide, 10 = sans insecticide, $\mathrm{I} 1$ = Insecticide 1 (Decis), $\mathrm{I} 2$ = Insecticide 2 (Systoate), $\mathrm{F}=$ Fongicide, F0 = sans fongicide, F1 = Fongicide 1 (Manèbe), F2 = Fongicide 2 (Peltar).

\section{RESULTATS}

\section{POPULATIONDE MOUCHE BLANCHE Bemisia tabaci (HOMOPTERA : ALEYRODIDAE)}

La figure 1 montre l'évolution des populations de Bemisia tabaci en fonction des traitements insecticides. En l'absence de traitement, un pic de pullulation correspondant à 300 insectes a été obtenu à $40 \mathrm{j}$ après repiquage (jar). En présence de deltaméthrine, deux pics ont été enregistrés, le premier plus petit à 26 jar et le second plus important à 40 jar, avec 100 insectes. Aussi, avec la combinaison diméthoate-deltaméthrine, un pic a été obtenu à 40 jar avec un nombre d'insectes inférieurs à 100.

\section{POPULATIONS DE CHENILLES}

Trois espèces de chenilles ont été recensées. II s'agit des noctuelles Helicoverpa armigera et Spodoptera sp. et de l'arpenteuse Plusia sp. La figure 2 montre l'évolution des populations de ces chenilles sur les parcelles non traitées. L'espèce Plusia sp. a atteint un pic de pullulation de 30 chenilles à 33 jar. A 61 jar, elle a été quasiment absente du champ. Helicoverpa armigera et Spodoptera sp. ont enregistré leurs pics de pullulation à 40 jar. L'effectif d' $H$. armigera a été de 80 individus, tandis que celui de Spodoptera sp. a été de 65. A 61 jar, 35 chenilles Spodoptera $s p$. et 45 chenilles $H$. armigera respectivement ont été encore présentes dans le champ.

\section{MALADIES DES PLANTES ET DES FRUITS}

Le tableau 2 présente les performances de la tomate en fonction des différents traitements insecticides et fongicides. Le taux d'avarie des fruits a été plus élevé (12 à $21 \%$ ) quand le traitement ne comprend pas d'insecticide et/ou de fongicide. Le témoin (sans insecticide et sans fongicide) a présenté le taux d'avarie le plus élevé $(23,7 \%)$. La combinaison deltaméthrinemanate 80 a permis d'enregistrer le plus faible taux d'avarie des tomates $(8,2 \%)$. Les maladies mycologiques observées en végétation ont révélé, principalement, les pourritures du collet, les dégâts d'alternariose et de stemphyliose. Le manèbe a semblé bien contrôler ces maladies. 


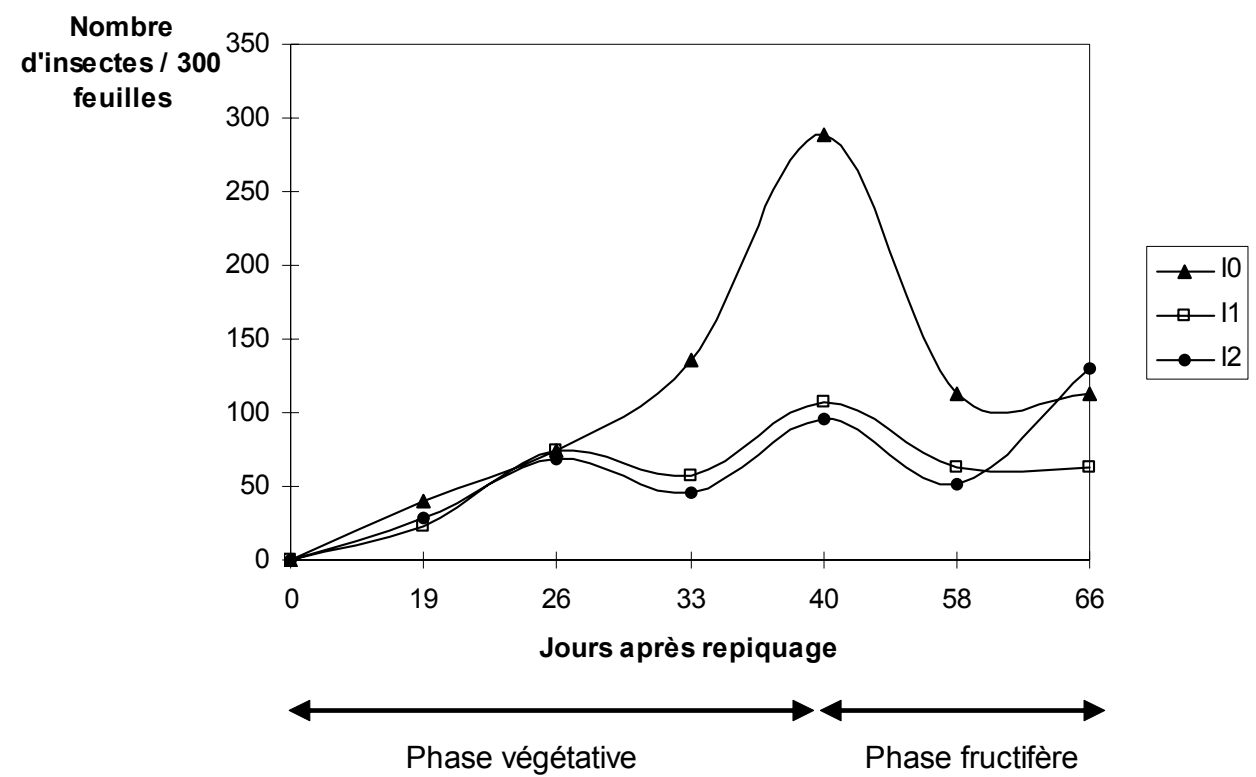

Figure 1 : Evolution de population de Bemisia tabaci en fonction des traitements insecticides. 10 : pas d'insecticide ; 11 : deltaméthrine ; 12 : diméthoate.

Changes in Bemisia tabaci population according to the insecticide treatments. 10 : no insecticide ; 11 : deltamethrine ; 12 : dimethoate.

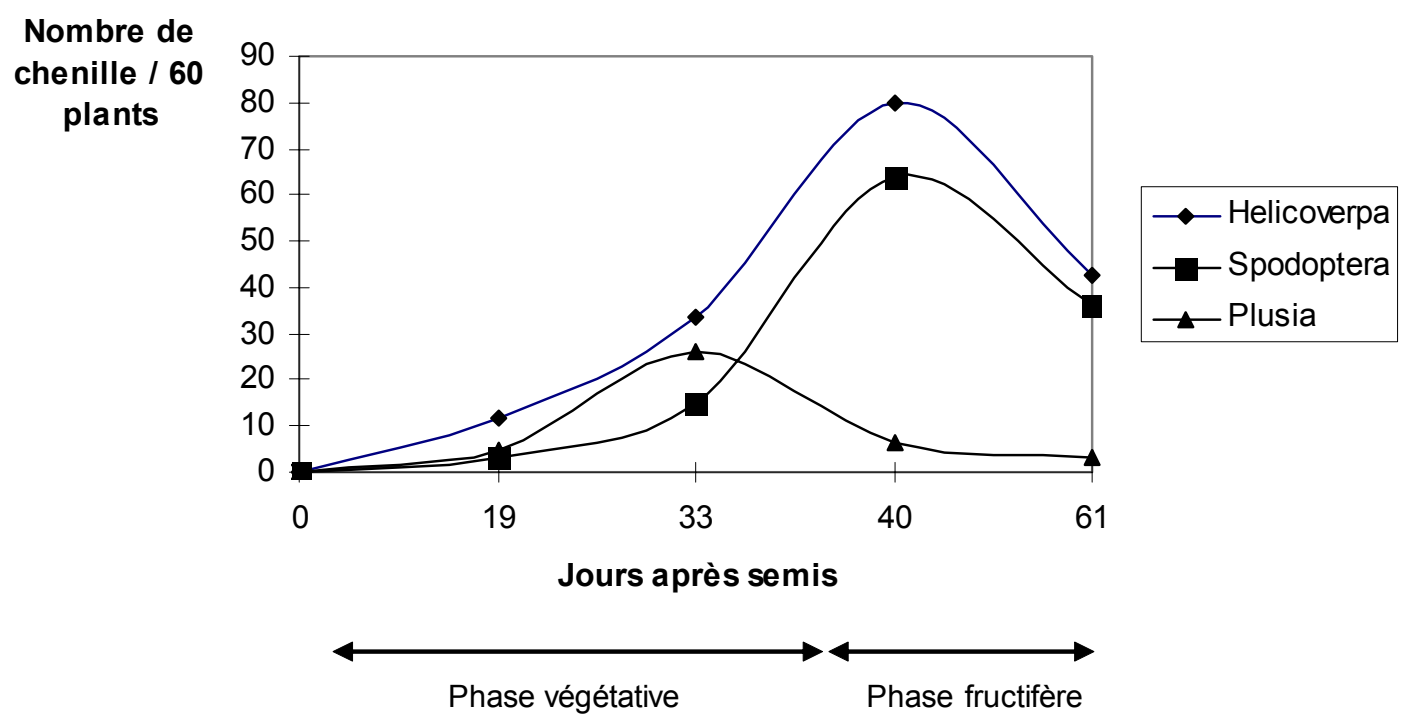

Figure 2 : Evolution des populations de chenilles sur les parcelles non traitées.

Changes in the caterpillar's population on the non-treated plots. 
Tableau 2 : Rendements de tomate en fonction des traitements phytosanitaires.

Tomato yield as a function of insecticide treatments.

\begin{tabular}{llccccc}
\hline \multicolumn{2}{c}{ Traitements } & $\begin{array}{c}\text { Rendement } \\
\text { total }(\mathrm{t} / \mathrm{ha})\end{array}$ & $\begin{array}{c}\text { Rendement } \\
\text { net }(\mathrm{t} / \mathrm{ha})\end{array}$ & $\begin{array}{c}\% \\
\text { d'avarie }\end{array}$ & $\begin{array}{c}\text { Gain net de } \\
\text { production }(\%) * *\end{array}$ & $\begin{array}{c}\text { Poids moyen } \\
\text { du fruit }(\mathrm{g})\end{array}$ \\
\hline Insecticides & Fongicides & & & & & \\
Sans & Sans & $21,50 \mathrm{c} *$ & $16,39 \mathrm{c}$ & 23.7 & 0 & $63.48 \mathrm{a}$ \\
Sans & manèbe & $31,87 \mathrm{~b}$ & $25,25 \mathrm{bc}$ & 20.7 & 54.05 & $62.82 \mathrm{a}$ \\
Sans & methylthiophanate & $27,47 \mathrm{bc}$ & $21,72 \mathrm{bc}$ & 20.8 & 32.51 & $60.19 \mathrm{a}$ \\
deltaméthrine & Sans & $29,38 \mathrm{bc}$ & $25,80 \mathrm{~b}$ & 12.0 & 57.71 & $63.49 \mathrm{a}$ \\
deltaméthrine & manèbe & $51,53 \mathrm{a}$ & $47,29 \mathrm{a}$ & 8.2 & 188.52 & $65.90 \mathrm{a}$ \\
deltaméthrine & methylthiophanate & $47,92 \mathrm{a}$ & $43 \mathrm{a}$ & 10.2 & 162.25 & $67.33 \mathrm{a}$ \\
diméthoate & Sans & $26,30 \mathrm{bc}$ & $21,65 \mathrm{bc}$ & 17.6 & 32.09 & $59.87 \mathrm{a}$ \\
diméthoate & manèbe & $48,68 \mathrm{a}$ & $43,64 \mathrm{a}$ & 10.3 & 166.25 & $65.54 \mathrm{a}$ \\
diméthoate & methylthiophanate & $48,52 \mathrm{a}$ & $43,49 \mathrm{a}$ & 10.3 & 165.34 & $65.94 \mathrm{a}$ \\
& & & & & & \\
\hline
\end{tabular}

*Les chiffres suivis de la même lettre, dans la même colonne, ne sont pas significativement différents au seuil de $5 \%$. S : significatif au seuil de $5 \%$; Ns : non significatif au seuil de $5 \%$. ${ }^{*}$ Le gain net de production est relatif à la parcelle sans insecticide ni fongicide.

\section{RENDEMENTS}

L'effet des traitements sur le rendement a été significatif. Les niveaux de rendement ont présenté une grande variation et sont supérieurs au témoin (16,4 t/ha). Ils ont varié de 21,7 avec peltar à $47,3 \mathrm{t} /$ ha pour la combinaison deltaméthrine-manate 80 (Tableau 2 et Figure 3). Les gains nets de production ont été de 160 à $180 \%$ comparés au témoin F0IO.
(Figure 4). Des différences significatives ont été également trouvées entre les variétés. L'analyse des résultats montre que la variété Avinash a donné le meilleur rendement. La deltaméthrine (insecticide) a permis d'obtenir les rendements nets les plus élevés. Les variétés Rossol et Avinash traitées aux fongicides et insecticides ont enregistré les meilleurs rendements nets ( 40 à 54 t/ha) (Tableau 3$)$.

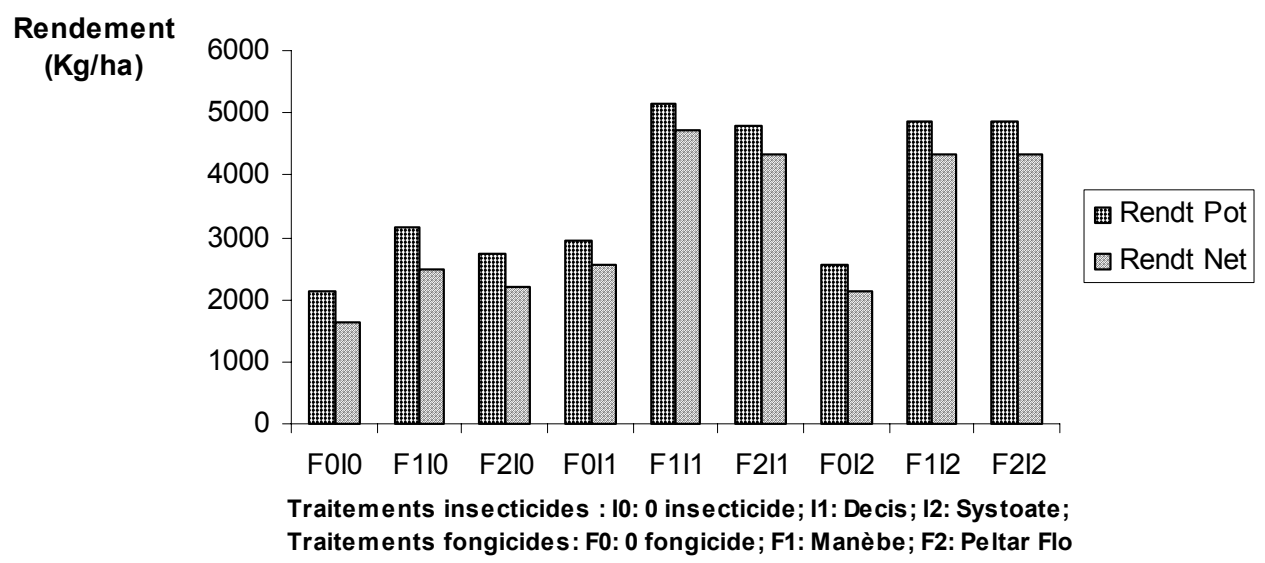

Figure 3 : Rendements nets de tomate obtenus en fonction des traitements combinés insecticidefongicide.

Net yield of tomato according to insecticide-fungicide treatments. 


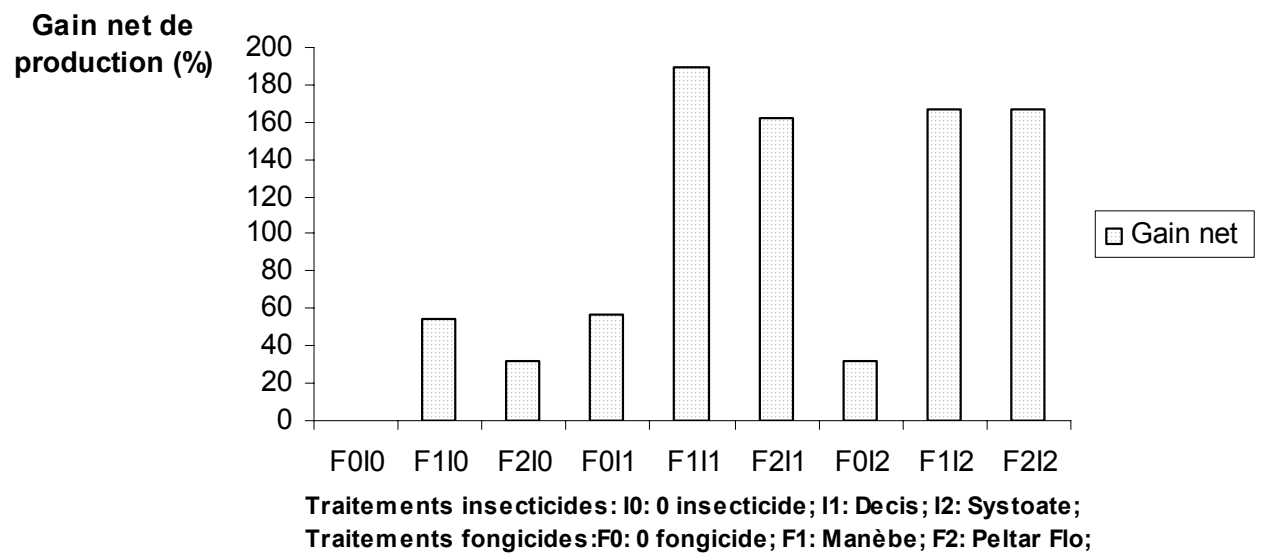

Figure 4 : Gain net de production suivant les traitements fongicide-insecticide.

Net gain of production according to of fungicide-insecticide treatments.

Tableau 3 : Rendements nets (t/ha) des variétés de tomate en fonction des traitements phytosanitaires.

Net yield of tomato varieties by treatment.

\begin{tabular}{llcccc}
\hline \multicolumn{2}{c}{ Traitement } & \multicolumn{4}{c}{ Variété } \\
\hline Insecticide & Fongicides & Rossol & Petomech & Avinash-2 & IDSA97-370 \\
\hline Sans & Sans & $21,05 \mathrm{f}-1^{*}$ & $16,76 \mathrm{~h}-\mathrm{j}$ & $16,29 \mathrm{~h}-\mathrm{j}$ & $11,47 \mathrm{j}$ \\
Sans & manèbe & $30,21 \mathrm{~b}-1$ & $14,91 \mathrm{l}-\mathrm{j}$ & $34,95 \mathrm{a}-1$ & $20,95 \mathrm{f}-\mathrm{j}$ \\
Sans & methylthiophanate & $23,43 \mathrm{e}-1$ & $20,03 \mathrm{~g}-\mathrm{j}$ & $23,67 \mathrm{e}-\mathrm{J}$ & $19,75 \mathrm{~h}-\mathrm{j}$ \\
deltaméthrine & Sans & $26,29 \mathrm{~d}-1$ & $28,41 \mathrm{~b}-\mathrm{j}$ & $27,64 \mathrm{c}-\mathrm{J}$ & $21,07 \mathrm{f}-\mathrm{j}$ \\
deltaméthrine & manèbe & $48,65 \mathrm{a}-\mathrm{b}$ & $41,20 \mathrm{a}-\mathrm{f}$ & $54,02 \mathrm{a}$ & $36,27 \mathrm{a}-\mathrm{h}$ \\
deltaméthrine & methylthiophanate & $42,83 \mathrm{a}-\mathrm{e}$ & $44,95 \mathrm{a}-\mathrm{d}$ & $47,97 \mathrm{a}-\mathrm{c}$ & $23,46 \mathrm{e}-\mathrm{j}$ \\
diméthoate & Sans & $22,63 \mathrm{e}-1$ & $20,75 \mathrm{f}-\mathrm{j}$ & $19,79 \mathrm{~h}-\mathrm{j}$ & $23,46 \mathrm{e}-\mathrm{j}$ \\
diméthoate & manèbe & $45,99 \mathrm{a}-\mathrm{d}$ & $34,98 \mathrm{a}-1$ & $50,85 \mathrm{a}$ & $42,74 \mathrm{a}-\mathrm{e}$ \\
diméthoate & methylthiophanate & $51,24 \mathrm{a}$ & $35,63 \mathrm{a}-1$ & $40,62 \mathrm{a}-\mathrm{g}$ & $46,50 \mathrm{a}-\mathrm{d}$ \\
\hline & & $\mathrm{S}$ & $\mathrm{S}$ & $\mathrm{S}$ & $\mathrm{S}$ \\
\hline
\end{tabular}

*Les chiffres suivis de la même lettre ne sont pas significativement au seuil de $5 \%$. S : significatif au seuil de $5 \%$. Ns : non significatif au seuil de $5 \%$

\section{DISCUSSION}

Les pics de pullulation de $B$. tabaci, vecteur des maladies virales comme l'enroulement foliaire, ont été atteints au $40^{\circ}$ jar correspondant, dans chacun des cas, au stade de fructification de la plante. Ces périodes de forte pullulation des populations de mouches pourraient résulter $\mathrm{du}$ bon développement des plants au champ. Les travaux d'Ohnesorge et al. (1980), Burban (1991) et de Urbino et al. (2005) ont montré que les populations de mouches blanches étaient importantes sur les jeunes feuilles et les plants au stade de développement végétatif. En effet, $B$. tabaci est polyphage et attaque plusieurs plantes cultivées ou non durant tout leur cycle de développement ; plus la plante croît, plus elle attire les insectes des plantes hôtes situées dans les environs immédiats de la parcelle de tomate. Les plants testés ont eu une végétation luxuriante qui a donc attiré beaucoup de mouches blanches.

De même, la population des mouches qui a été beaucoup plus élevée sur les parcelles non traitées $(I 0)$ que sur les parcelles traitées à la deltaméthrine (I1) et/ou au diméthoate (I2), montre l'efficacité de ces produits contre cet insecte.

Les niveaux de populations de chenilles ont varié suivant le stade phénologique de la plante, d'une part, et les traitements insecticides, d'autre part. La comparaison des populations de chenilles des parcelles non traitées a révélé que les noctuelles (H. armigera. et Spodoptera sp.) ont été plus nombreuses au moment de la fructification alors que les arpenteuses ont été 
plus importantes en début de floraison ; en effet, bien que phyllophages, $H$. armigera et Spodoptera sp. soient surtout carpophages. De plus, les adultes (les papillons) ont été attirés par les fleurs, d'où les fortes pullulations pendant la fructification. La baisse de la population d'insectes en phase de maturation des fruits pourrait s'expliquer par la nymphose amorcée par les chenilles. Contrairement aux noctuelles, les arpenteuses (Plusia sp.) étant essentiellement phyllophages, leurs populations ont été plus importantes en phase végétative, d'où le pic observé au 30e jar. Selon Tidon et al., (1992) les variations naturelles des populations pourraient masquer l'effet des insecticides. En effet, Pavis (2006) a confirmé que les populations de ces ravageurs varient aussi selon les zones et les périodes de culture.

Les dégâts mycologiques sur les plants ont été importants. Les taux d'attaque des champignons ont été plus élevés sur parcelles non traitées que traitées. La deltaméthrine (insecticide) a mieux protégé les fruits, avec seulement $3 \%$ de pertes dues aux insectes. Ce produit a été efficace contre les chenilles. En effet, il s'agit d'une pyréthrine recommandée contre les noctuelles (Fondio et al., 2007). Le manèbe a également bien contrôlé les attaques dues aux champignons avec une perte de fruits de 2 à $3 \%$.

Au niveau de la sensibilité aux attaques, la variété, IDSA 97-370 a été plus sensible, alors que Petomech a subi très peu de dégâts physiologiques. Les variétés Rossol et Petomech ont semblé être moins sujettes aux attaques de champignons.

L'application simultanée de fongicide (manèbe) et d'insecticide (deltaméthrine) a permis d'obtenir les meilleurs rendements. Ce qui montre que le fongicide et l'insecticide en application simultanée sont nécessaires pour accroître le rendement de la tomate. Les rendements des variétés Rossol et Avinash-2 comparés à ceux obtenus par les autres variétés de tomate dans la zone d'étude, ont été élevés (Kouamé et al., 1997 ; Kouamé et al., 2002). Les travaux de N'zi et al. (2000) à Bouaké (Foro-
Foro) ont rapporté des rendements maxima de 32 t/ha pour des variétés de tomate. Ces rendements sont nettement inférieurs à ceux enregistrés dans cette étude. Van der Vossen et al., (2004) ont aussi rapporté des rendements de tomate de moins de 15 t/ha dans de nombreux pays d'Afrique subsaharienne. Les performances élevées de la variété Avinash-2 résulteraient de l'expression de son caractère hybride, avec une tolérance à plusieurs ravageurs ou maladies. En effet, les performances de la tomate ont varié en fonction des facteurs agro écologiques. Plus les facteurs biotiques (Pavis, 2006) et abiotiques sont maîtrisés, plus les rendements obtenus par les variétés sont élevés (Hanafi, 2000). Huat (2006) confirme ces résultats sur les facteurs qui limitent le rendement de la tomate au nord du Sénégal. L'efficacité des traitements phytosanitaires jouerait ainsi, un rôle essentiel sur la production de la tomate par la réduction du niveau de pullulation des ravageurs et des dégâts causés par ceux-ci.

\section{CONCLUSION}

L'efficacité des combinaisons d'insecticides et de fongicides a été observée sur 4 variétés de tomate. Les parcelles traitées à la deltaméthrine et au manèbe ont enregistré les plus faibles pertes en fruits de tomate. Cette utilisation simultanée a donné un gain net de production de $88 \%$. Ainsi, l'utilisation de la deltaméthrine et du diméthoate a été efficace contre la mouche blanche et les chenilles. Le manèbe a mieux contrôlé les maladies. L'application des pesticides a permis d'obtenir des rendements deux à trois fois plus élevés que les témoins. Toutefois, la maîtrise des doses des produits et les périodes d'application sont importante pour une récolte saine, sans résidus toxique. La protection de l'environnement contre l'abus des pesticides, étant un sujet d'actualité, il apparait opportun d'approfondir la présente étude en l'inscrivant dans une perspective de lutte intégrée qui permette de réduire les traitements chimiques au profit des méthodes alternatives. 


\section{REMERCIEMENT}

Cette étude a été réalisée dans le cadre de la convention IDESSA/TRITURAF pour le développement de la culture de la tomate d'industrie. Les auteurs expriment leur gratitude aux promoteurs de la convention et dédient cet article au premier auteur, feu Aristide Kouakou Adjé, entomologiste, trop tôt disparu.

\section{REFERENCES}

Burban C. 1991. Structuration des populations chez un insecte polyphage : Bemisia tabaci (Genn.) (Homoptera: Aleyrodidae). Thèse de doctorat, Université de Montpellier II, Sciences et techniques du Languedoc, France. $103 \mathrm{p}$.

Conover R. A. and N. R. Gerhold. 1981. Mixtures of copper and maneb or man-cozeb for control of bacterial spot of tomato and their capability for control of fungus diseases. Proc. Florida State Hort. Soc. 94: 154 - 156.

Fondio L., Djidji A. H., Kouamé C. N., Aïdara S. et N. Hala. 2007. Bien cultiver le gombo en Côte d'Ivoire. Fiche Technique CNRA/CTA, 4 p.

Hanafi A. 2000. La mouche blanche et le virus des feuilles en cuillère de la tomate (Tylcv). In Transfert de technologie en Agriculture. $N^{\circ}$ 73. MADREF/DERD. PNTTA. Rabat Maroc. 4 p.

Huat J. 2006. Facteurs limitatifs du rendement de la tomate industrielle en périmètres irrigués au Nord Sénégal. Cah. Agric. 15 (3): $293-300$

Kouamé C. N., Djidji A. H. et Fondio L. 1997. Rapport d'achèvement du projet de développement de la culture de la tomate d'industrie en région Centre et Centre-Nord de la Côte-d'Ivoire. IDESSA, Bouaké. 30 p.

Nechadi S., Benddine F., Moumen A. et M. Kheddam. 2002. Etat des maladies virales de la tomate et stratégie de lutte en Algérie. Bulletin OEPP. Blackwell Synergy. Vol. $32 \mathrm{p}$ 21. Doi : 10.

Ohnesorge B., Sharaf N. and T. Allawi. 1980. Population studies on the tobacco whitefly Bemisia tabaci Genn. (Homoptera : Aleyrodidae) during the winter season.
Part I. Journal of Applied Entomology. 90 (3) : $226-232$.

Ohnesorge B., Sharaf N. and T. Allawi. 1981. Population studies on the tobacco whitefly Bemisia tabaci Genn. (Homoptera : Aleyrodidae) during the winter season. Part II. Journal of Applied Entomology. $92(2)$ : 127 - 136.

Open R. T., Green S. K., Talker N. S. and J. T. Chen. 1989. Genetic improvement of tomato for adaptability to the tropics : Progress and future prospects. Proc. of the International Symposium on integrated management practices. AVRDC, Tainan, Taiwan. 78 - 85.

Pavis C. 2006. Structure des populations des bioagresseurs. Une grande variabilité spatiale et temporelle des populations des bioagresseurs et d'auxiliaires. INRA. Antilles-Guyane. $1 \mathrm{p}$.

Philouze J. et H. Laterrot. 1992. La tomate, In A. Gallais et $\mathrm{H}$. Bannerot (Eds.). Amélioration des espèces végétales cultivées. INRA, Paris. 379 - 391

Pohronezny (K.) and R. B. Volin. 1983. The effect of bacterial spot on yield and quality of fresh market tomato. Hortsci. $18: 69$ - 70.

SAS. Statistical Analysis System. 1986. SAS Institute Inc, Cary, NC.

Somodi G. C., Jones J. B. and S. W. Scott. 1989. Relation ship of lesion development on resistant and susceptible tomatoes to internal populations of Xanthomonas campestris pv. Vesicatoria. Proceedings of the International symposium on Integrated Management practices. AVRDC. Tainan, Taïwan. 208 - 212.

Tidon-Sklorz R. and F. M. Sene. 1992. Vertical and temporal distribution of Drosophila (Diptera, Drosophilidae) species in wooded area in the state of São Paulo, Brazil. Rev Bras Biol $52: 311-317$

Urbino C., M. L. Caruana., Pavis C., La Fortune D., Boissot N. et J. Dintinger. 2005. Protection intégrée de la tomate contre les maladies à begomovirus. CIRAD Flhor. France. $1 \mathrm{p}$.

Van der Vossen Y. A. M., Nono-Womdim R. et C. M. Messiaen. 2004. Lycopersicum esculentum Mill. Fiche Protabase. Gruben G. J. H. and O. A. Denton. (Eds). PROTA (Plant Resources of Tropical Africa) Wageningen, Pays-Bas. 668 p. 